\title{
若尔盖高原湿地不同微地貌区甲烷排放通量特征
}

\author{
周文昌 ${ }^{1,2}$ 崔丽娟 ${ }^{*}$ 王义飞 ${ }^{1}$ 李 伟 ${ }^{1}$ 康晓明 ${ }^{1}$ \\ ${ }^{1}$ 中国林业科学研究院湿地研究所湿地生态功能与恢复北京市重点实验室, 北京 100091 ; ${ }^{2}$ 湖北省林业科学研究院, 武汉 430075
}

\begin{abstract}
摘 要 若尔盖高原是我国泥炭沼泽湿地的主要分布区、青藏高原的主要甲烷 $\left(\mathrm{CH}_{4}\right)$ 排放中心。为了研究湿地微地貌环境对 高原湿地 $\mathrm{CH}_{4}$ 排放通量的影响, 2014年5-10月, 采用静态箱和快速温室气体分析仪原位测量若尔盖高原湖滨湿地3种泥炭沼泽 5 种微地貌环境下的 $\mathrm{CH}_{4}$ 排放通量特征。结果表明: (1)常年性淹水泥炭湿地洼地 (P-hollow)和草丘(P-hummock)生长季平均 $\mathrm{CH}_{4}$ 排放通量为 68.48 和 $40.32 \mathrm{mg} \cdot \mathrm{m}^{-2} \cdot \mathrm{h}^{-1}$, 季节性淹水的泥炭湿地洼地(S-hollow)和草丘(S-hummock)平均 $\mathrm{CH}_{4}$ 排放通量为2.38和 $0.63 \mathrm{mg} \cdot \mathrm{m}^{-2} \cdot \mathrm{h}^{-1}$, 而无淹水平坦地 $(\mathrm{Lawn})$ 平均 $\mathrm{CH}_{4}$ 排放通量为 $3.68 \mathrm{mg} \cdot \mathrm{m}^{-2} \cdot \mathrm{h}^{-1}$; (2)湿地 5 种微地貌区 $\mathrm{CH}_{4}$ 排放通量为 $(23.10 \pm$ 30.28) $\mathrm{mg} \cdot \mathrm{m}^{-2} \cdot \mathrm{h}^{-1}$ (平均值 \pm 标准偏差)), 变异系数为 $131 \%$ 。分析显示这 5 种微地貌区 $\mathrm{CH}_{4}$ 排放通量的平均值与其水位深度平均 值存在显著的线性正相关关系 $\left(R^{2}=0.919, p<0.01\right)$, 表明水位深度是控制湿地微地貌区 $\mathrm{CH}_{4}$ 排放通量空间变化的主要因子; (3) P-hummock、P-hollow和S-hummock的 $\mathrm{CH}_{4}$ 排放通量存在显著的季节变化, Lawn和S-hollow无明显的季节性变化, 但5种微 地貌区在夏季或秋季均观测到 $\mathrm{CH}_{4}$ 排放通量峰值, 其影响因子可能与水位深度、土壤温度和凋落物输入密切相关; (4) P-hollow可能时常发生冒泡式 $\mathrm{CH}_{4}$ 排放, 这可能导致过去低估了若尔盖高原湿地的 $\mathrm{CH}_{4}$ 排放量。
\end{abstract}

关键词 甲烷排放通量; 草丘; 洼地; 平坦地; 若尔盖高原湿地

引用格式: 周文昌, 崔丽娟, 王义飞, 李伟, 康晓明 (2016). 若尔盖高原湿地不同微地貌区甲烷排放通量特征. 植物生态学报, 40, 902-911. doi: 10.17521/cjpe.2016.0029

\section{Characteristics of methane emission fluxes in the Zoigê Plateau wetland on microtopography}

\section{ZHOU Wen-Chang ${ }^{1,2}$, CUI Li-Juan ${ }^{1 *}$, WANG Yi-Fei ${ }^{1}$, LI Wei ${ }^{1}$, and KANG Xiao-Ming ${ }^{1}$}

${ }^{1}$ Beijing Key Laboratory of Wetland Services and Restoration, Institute of Wetland Research, Chinese Academy of Forestry, Beijing 100091, China; and ${ }^{2}$ Hubei Academy of Forestry, Wuhan 430075, China

\section{Abstract}

Aims The Zoigê Plateau, as a very important wetland distribution region of China, was the major methane $\left(\mathrm{CH}_{4}\right)$ emission center of the Qinghai-Xizang Plateau. The objective of this study is to study the effects of microtopographic changes on $\mathrm{CH}_{4}$ emission fluxes from five plots across three marshes in the littoral zone of the Zoigê Plateau wetland.

Methods $\mathrm{CH}_{4}$ emission fluxes were measured in five plots across three marshes in Zoigê Plateau wetland using the closed chamber method and Fast Greenhouse Gas Analyzer from May to October in 2014.

Important findings During the growing season, mean $\mathrm{CH}_{4}$ emission fluxes from the permanently flooded hollow (P-hollow) and hummock (P-hummock) in the Zoigê Plateau wetland were 68.48 and $40.32 \mathrm{mg} \cdot \mathrm{m}^{-2} \cdot \mathrm{h}^{-1}$, while mean $\mathrm{CH}_{4}$ emission fluxes from the seasonally flooded hollow (S-hollow) and hummock (S-hummock) were 2.38 and $0.63 \mathrm{mg} \cdot \mathrm{m}^{-2} \cdot \mathrm{h}^{-1} \cdot \mathrm{CH}_{4}$ emission fluxes from non-flooded lawn was $3.68 \mathrm{mg} \cdot \mathrm{m}^{-2} \cdot \mathrm{h}^{-1}$. Mean $\mathrm{CH}_{4}$ emission fluxes from five plots across three sites was $23.10 \mathrm{mg} \cdot \mathrm{m}^{-2} \cdot \mathrm{h}^{-1}$, with a standard deviation of 30.28 $\mathrm{mg} \cdot \mathrm{m}^{-2} \cdot \mathrm{h}^{-1}$ and the coefficient of variation was $131 \%$. We also found that there was a significant and positive correlation between mean $\mathrm{CH}_{4}$ emission fluxes and mean water table depth in the five plots across three sites $\left(R^{2}=\right.$ 0.919, $p<0.01$ ), indicating that water table depth was controlling the spatial variability of $\mathrm{CH}_{4}$ emission fluxes from the Zoigê Plateau wetland on microtopography. $\mathrm{CH}_{4}$ emission fluxes in the P-hollow, P-hummock, and S-hummock showed an obvious seasonal pattern, which was not observed in the lawn and S-hollow. However, $\mathrm{CH}_{4}$ emission peaks were observed in all the plots during summer and/or autumn, which could be closely related to the water table depth, soil temperature, and the magnitude of litter mass. In addition, we found that the $\mathrm{CH}_{4}$ emission flux in the P-hollow was much higher than the other four plots in the Zoigê Plateau wetland, suggesting

收稿日期Received: 2016-01-17 接受日期Accepted: 2016-05-09

* 通信作者Author for correspondence (E-mail: lkyclj@126.com) 
that $\mathrm{CH}_{4}$ in the P-hollow could be often transported to the surface by ebullition and $\mathrm{CH}_{4}$ emission from the Zoigê Plateau wetland may be under estimated in the past.

Key words $\mathrm{CH}_{4}$ emission flux; hummock; hollow; lawn; Zoigê Plateau wetland

Citation: Zhou WC, Cui LJ, Wang YF, Li W, Kang XM (2016). Characteristics of methane emission fluxes in the Zoigê Plateau wetland on microtopography. Chinese Journal of Plant Ecology, 40, 902-911. doi: 10.17521/cjpe.2016.0029

甲烷 $\left(\mathrm{CH}_{4}\right)$ 是一种重要的温室气体, 它比 $\mathrm{CO}_{2}$ 活 跃, 其单分子的增温效应为 $\mathrm{CO}_{2}$ 的 28 倍 (IPCC, 2013)。由于人类活动的影响, 大气中的 $\mathrm{CH}_{4}$ 含量已 从1750年的 $0.722 \mu \mathrm{mol} \cdot \mathrm{mol}^{-1}$ 上升到 2011 年的 1.803 $\mu \mathrm{mol} \cdot \mathrm{mol}^{-1}$, 升高了约2.5倍(IPCC, 2013)。虽然在 1999-2006年大气中的 $\mathrm{CH}_{4}$ 含量趋于稳定, 但从 2007年开始, 大气 $\mathrm{CH}_{4}$ 含量再次升高(Rigby et al., 2008; Kirschke et al., 2013), 这主要是由于湿地、稻 田和生物质燃烧排放的 $\mathrm{CH}_{4}$ 增加(Chen \& Prinn, 2006; Kirschke et al., 2013)。因此, $\mathrm{CH}_{4}$ 的源/汇问题 仍是目前研究的热点, 加强 $\mathrm{CH}_{4}$ 源/汇问题的研究对 于认识和预测 $\mathrm{CH}_{4}$ 在全球气候变暖过程中的作用具 有重要意义。

自然湿地是大气 $\mathrm{CH}_{4}$ 的重要排放源, 年排放量 为177-284 $\mathrm{Tg}\left(1 \mathrm{Tg}=10^{12} \mathrm{~g}\right)$, 约占全球 $\mathrm{CH}_{4}$ 排放量 的26\%-42\% (IPCC, 2013), 排放量的不确定, 一是 由于不同湿地的 $\mathrm{CH}_{4}$ 排放存在时空变化格局 (Whalen \& Reeburgh, 1992; Huttunen et al., 2003; Inubushi et al., 2005; Chen et al., 2008; Glagolev et al., 2011; 黄璞祎等, 2011; McEwing et al., 2015; Song et al., 2015), 二是因为气候变化(IPCC, 2013; Munir \& Strack, 2014)。湿地 $\mathrm{CH}_{4}$ 排放经由厌氧条件 下产 $\mathrm{CH}_{4}$ 菌生成 $\mathrm{CH}_{4}$ 和需氧条件下氧化 $\mathrm{CH}_{4}$ 菌氧化 $\mathrm{CH}_{4}$ 两种微生物过程, 以扩散、冒泡和植物传输 3 个 过程排放 $\mathrm{CH}_{4}$ (Le Mer \& Roger, 2001; 王智平等, 2003; Lai, 2009; McEwing et al., 2015)。这3个过程受 许多环境因子(温度、水位深度、底物活性和植物类 型)影响 (Mikkelä et al., 1995; 丁维新和蔡祖聪, 2002; Inubushi et al., 2005; McEwing et al., 2015; Wei et al., 2015)。由于湿地(如泥炭地)形成了多种生 态系统及其系统内异质性地貌(Lai, 2009; Glagolev et al., 2011; Munir \& Strack, 2014; Song et al., 2015; Wei et al., 2015), 不同水位深度条件下, 植被和土 壤温度具有差异, 进而导致 $\mathrm{CH}_{4}$ 排放通量存在时空 变化(Dise, 1993; 王智平等, 2003; Hirota et al., 2004; Wei et al., 2015)。目前, 国外有关泥炭地微地
貌区 $\mathrm{CH}_{4}$ 排放的研究较多(Dise, 1993; Mikkelä et al., 1995; Waddington \& Roulet, 1996; Glagolev \& Shnyrev, 2008; Kalyuzhnyi et al., 2009; Munir \& Strack, 2014), 而国内有关这方面的研究鲜有报道 (Wei et al., 2015)。因此, 进一步研究有助于人们深 刻理解不同空间湿地 $\mathrm{CH}_{4}$ 排放对环境变化的响应机 制和精确预算区域湿地 $\mathrm{CH}_{4}$ 排放量。

若尔盖高原 $\left(101.60^{\circ}-103.50^{\circ} \mathrm{E}, 32.33^{\circ}-34.00^{\circ}\right.$ $\mathrm{N}$, 平均海拔为 $3400 \mathrm{~m}$ ) 泥炭沼泽面积约为 4038 $\mathrm{km}^{2}$, 是我国面积最大的高原泥炭沼泽分布区 (王德 宣等, 2002), 也是青藏高原东部边缘的 $\mathrm{CH}_{4}$ 排放源 (Jin et al., 1999)。近10年来, 国内专家研究若尔盖高 原泥炭沼泽湿地 $\mathrm{CH}_{4}$ 排放特征(王德宣等, 2002; Ding et al., 2004; Hirota et al., 2004; Chen et al., 2008; 王德宣, 2010; 李丽等, 2011; Song et al., 2015), 取得了一定的成果, 为理解高原湿地碳循环 和 $\mathrm{CH}_{4}$ 排放机理提供了一定理论基础。然而, 若尔 盖高原泥炭沼泽微地貌区(草丘和洼地) $\mathrm{CH}_{4}$ 排放的 时空变化格局鲜有报道。Wei等(2015)报道了青藏高 原两种海拔高度(4 758和4 $320 \mathrm{~m}$ ) 的湿地微地貌区 (草丘和洼地) $\mathrm{CH}_{4}$ 排放特征, 结果表明其影响因子 较多, 存在时间和空间上的差异, 区域 $\mathrm{CH}_{4}$ 排放量 仍存在较大的不确定性, 这将不利于我们深刻理解 高原湿地 $\mathrm{CH}_{4}$ 排放对环境变化和气候变化的响应机 制, 以及精确预算我国高原湿地 $\mathrm{CH}_{4}$ 排放量。

\section{1 研究区概况和研究方法}

\section{1 研究区概况}

本研究地点 $\left(33.92^{\circ} \mathrm{N}, 102.82^{\circ} \mathrm{E}\right.$, 海拔为 3441 $\mathrm{m}$ )位于若尔盖湿地自然保护区。若尔盖湿地2008年 被列入《湿地公约》的国际重要湿地名录。该地属 于寒温带湿润气候, 11 月至次年4月受西伯利亚和蒙 古的冷空气控制, 5至10月受西南季风控制, 年平均 气温为 $1{ }^{\circ} \mathrm{C}$, 最暖月7月平均气温为 $10.7{ }^{\circ} \mathrm{C}$, 最冷 月1月平均气温-10.3 ${ }^{\circ} \mathrm{C}$ (王智平等, 2003; Ding et al., 2004)。年降水量650 mm, 降水集中在6-9月, 相 
对湿度78\% (王智平等, 2003)。

研究地点位于花湖湖泊边缘, 有 3 种水位深度 的泥炭湿地: 常年性淹水、季节性淹水和地表无淹 水。在这3种泥炭湿地, 常年性淹水泥炭湿地位于湖 泊边缘, 季节性淹水泥炭湿地位于湖泊外围, 地表 无淹水泥炭湿地位于前两个样地之间的过渡带。常 年性淹水和季节性淹水泥炭湿地均形成了微地貌 草丘(hummock)和洼地(hollow), 这两种微地貌面积 所占比例约为 $55 \%$ 和 $45 \%$ 。共有 5 种微地貌, 其植物 类型如下: (1)常年性淹水草丘(P-hummock)主要植 物为木里薹草(Carex muliensis); (2)常年性淹水洼地 (P-hollow) 主要植物为沉水植物小眼子菜(Potamogeton pusillus)和狸藻(Utricularia vulgaris), 伴生稀 疏的木里薹草; (3)季节性淹水草丘(S-hummock)主 要植物为西藏嵩草(Kobresia tibetica), 伴生具刚毛 荣荠(Eleocharis valleculosa)、蕨麻(Potentilla anserina)和花葶驴蹄草(Caltha scaposa); (4)季节性淹 水洼地(S-hollow)主要植物为木里薹草; (5)过渡带 平坦地(lawn)主要植物为西藏嵩草和花葶驴蹄草。 土壤类型为泥炭沼泽土, 土壤理化性质见表1。

\section{2 研究方法}

\subsection{1 样地设置}

2014年4月下旬, 在花湖湖滨湿地5种微地貌区 各建立3个标准样地 $(20 \mathrm{~m} \times 20 \mathrm{~m})$, 在5种微地貌区 各安装静态箱底座 3 个 $(n=3)$, 共 15 个。底座由不锈 钢制作 (规格为 $50 \mathrm{~cm} \times 50 \mathrm{~cm} \times 20 \mathrm{~cm}$ ), 底座上口 四周有 $5 \mathrm{~cm}$ 高度的水槽, 下口插入土壤 $15 \mathrm{~cm}$, 底 座永久保留在实验地土壤中。同时, 在常年性淹水 样地, 用直径 $15 \mathrm{~cm}$ 的原木搭建栈道, 通过铁钉固 定，防止取样时人为对土壤的干扰。

\subsection{2 $\mathrm{CH}_{4}$ 气体测量}

通过静态箱法(Chen et al., 2008; McEwing et $a l ., 2015)$ 采集 $\mathrm{CH}_{4}$ 。静态箱由底座、中箱 $(50 \mathrm{~cm} \times 50$ $\mathrm{cm} \times 50 \mathrm{~cm}$ ) 和顶箱 $(50 \mathrm{~cm} \times 50 \mathrm{~cm} \times 50 \mathrm{~cm}$ ) 组成(孙 晓新等, 2009)。常年性淹水P-hollow采用底座、中 箱和顶箱测量 $\mathrm{CH}_{4}$ 气体, 其他微地貌区采用底座和 顶箱测量 $\mathrm{CH}_{4}$ 气体。中箱和顶箱由薄的铝材料制成, 中箱上口四周有 $5 \mathrm{~cm}$ 高度的水槽, 防止气体泄露, 为了使箱内温度稳定, 中箱和顶箱外包装塑料泡 沫, 顶箱内部有 2 个 $5 \mathrm{~cm} \times 5 \mathrm{~cm}$ 的风扇, 顶箱上部 中央附有直径为 $2 \mathrm{~cm}$ 的 2 个橡皮塞小圆孔，连接快 速温室气体分析仪器(Model GGA-24EP, Los Gatos Research, San Jose, USA)的2根附有橡皮塞的透明 导气管,长度 $4 \mathrm{~m}$ (内径为 $4 \mathrm{~mm}$ )(Mastepanov et al., 2008; McEwing et al., 2015), 仪器通过12 V蓄电池 供电, 数据采集设置为 $1 \mathrm{~Hz}$ (Mastepanov et al., 2008)。启动仪器后, 测量 $\mathrm{CH}_{4}$ 排放通量时, 静态箱 与底座水槽密闭后, 密闭箱内空气进入分析仪, 并 通过2根透明导气管来回在分析仪器内无损坏地循 环分析 $\mathrm{CH}_{4}$ 浓度变化。每次气体测量之前, 在底座 水槽注满水, 启动仪器, 等待仪器启动显示的顶箱 内部大气 $\mathrm{CH}_{4}$ 浓度稳定为当地环境气体 $\mathrm{CH}_{4}$ 浓度 $\left(8.04 \times 10^{-8} \mathrm{~mol} \cdot \mathrm{L}^{-1}\right)$ 时, 将静态箱扣在底座或中箱 上, 密闭测量3-5 min, 然后揭开静态箱置于开放状 态, 约为 $2 \mathrm{~min}$, 然后密闭测量下一个静态箱底座, 循环操作以上过程。测量时间为2014年5-10月北京 时间 9:00-11:30, 观测频率为每月 2次。 $\mathrm{CH}_{4}$ 排放通 量是以封闭箱内顶部的 $\mathrm{CH}_{4}$ 浓度随时间变化的直线 斜率计算(Mastepanov et al., 2008; McEwing et al., 2015), 回归方程决定系数 $R^{2} \geqslant 0.90$; 当 $R^{2}<0.90$ 时,

表1 若尔盖高原湿地5种微地貌土壤理化性质(平均值土标准偏差)

Table 1 Soil physical and chemical properties from the Zoigê Plateau wetland on five microtopography (mean $\pm S D$ )

\begin{tabular}{llllll}
\hline 样点 Plot & $\begin{array}{l}\mathrm{pH} \\
(0-10 \mathrm{~cm} \text { depth) }\end{array}$ & $\begin{array}{l}\text { 土壤有机碳含量 } \\
\begin{array}{l}\text { Soil organic carbon content } \\
\left(0-30 \mathrm{~cm} \mathrm{depth)}\left(\mathrm{g} \cdot \mathrm{kg}^{-1}\right)\right.\end{array}\end{array}$ & $\begin{array}{l}\text { 土壤容重 } \\
\text { Bulk density } \\
(0-30 \mathrm{~cm} \text { depth })\left(\mathrm{g} \cdot \mathrm{cm}^{-3}\right)\end{array}$ & $\begin{array}{l}\text { 总氮 } \\
\text { Total nitrogen } \\
\left(0-10 \mathrm{~cm} \mathrm{depth)}\left(\mathrm{g} \cdot \mathrm{kg}^{-1}\right)\right.\end{array}$ & $\begin{array}{l}\text { 地上生物量 } \\
\text { Aboveground biomass } \\
\left(\mathrm{g} \cdot \mathrm{m}^{-2}\right)\end{array}$ \\
\hline P-hummock & $7.6 \pm 0.1$ & $210.38 \pm 47.29$ & $0.29 \pm 0.09$ & $15.20 \pm 3.50$ & $127.16 \pm 8.11$ \\
P-hollow & & & & $279.34 \pm 35.54$ \\
Lawn & $7.5 \pm 0.3$ & $143.21 \pm 10.03$ & $0.52 \pm 0.08$ & $18.05 \pm 0.00$ & $142.28 \pm 95.61$ \\
S-hummock & $7.5 \pm 0.2$ & $151.74 \pm 74.15$ & $0.40 \pm 0.16$ & $8.91 \pm 3.85$ & $189.74 \pm 72.79$ \\
S-hollow & & & & & $194.01 \pm 50.07$
\end{tabular}

Lawn，常年性淹水与季节性淹水点之间的过渡带平坦地; P-hollow，常年性淹水洼地; P-hummock，常年性淹水草丘; S-hollow，季节性淹水洼地; S-hummock, 季节性淹水草丘。

Lawn, transitional zones between permanently flooded and seasonally flooded sites; P-hollow, permanently flooded hollow; P-hummock, permanently flooded hummock; S-hollow, seasonally flooded hollow; S-hummock, seasonally flooded hummock. 
数据不作为 $\mathrm{CH}_{4}$ 排放通量计算, 其比例为 $3.8 \%$ (包 含 P-hollow 的两个瞬时值过大 (592.44 和 327.82 $\left.\mathrm{mg} \cdot \mathrm{m}^{-2} \cdot \mathrm{h}^{-1}\right)$, 尽管 $\left.R^{2}>0.90\right) 。 \mathrm{CH}_{4}$ 排放通量的计算公 式(孙晓新等, 2009; McEwing et al., 2015)如下:

$$
F=\frac{d_{c}}{d_{t}} \times \frac{M}{V_{0}} \times \frac{P}{P_{0}} \times \frac{T}{T_{0}} \times \frac{V}{A}
$$

式中: $F$ 为 $\mathrm{CH}_{4}$ 排放通量 $\left(\mathrm{mg} \cdot \mathrm{m}^{-2} \cdot \mathrm{h}^{-1}\right) ; M$ 为被测气体 的摩尔质量, $V$ 为箱内空气体积; $A$ 为静态箱底面积 $\left(0.25 \mathrm{~m}^{2}\right) ; d_{d} / d_{t}$ 代表 $\mathrm{CH}_{4}$ 浓度随时间变化的直线斜 率; $V_{0} 、 T_{0}$ 和 $P_{0}$ 分别为标准状态下的 $\mathrm{CH}_{4}$ 气体摩尔体 积 $\left(22.4 \mathrm{~L} \cdot \mathrm{mol}^{-1}\right)$ 、空气绝对温度 $(273.15 \mathrm{~K})$ 和气压 $(1013.25 \mathrm{hPa})$; $P$ 为采样地点的气压; $T$ 为采样时箱 内的绝对温度。

测量 $\mathrm{CH}_{4}$ 排放通量时, 采用数字温度计测量 6 种 深度(5、10、15、20、30和 $45 \mathrm{~cm}$ ) 的土壤温度。通过 在静态箱附近挖井约70 cm测量土壤水位深度, 而常 年性淹水点直接测量地表水位深度值, 2014年8月中 旬测量地上生物量, 采集 3 个重复样方面积为 $50 \mathrm{~cm}$ $\times 50 \mathrm{~cm}$ 的地上生物量, 运输到实验室恒温箱 $70{ }^{\circ} \mathrm{C}$ 烘 干至恒质量, 称量。另外, 在3种类型的泥炭沼泽中 取0-30 cm深度土壤测量土壤理化性质(表1)。

\subsection{3 数据统计}

采用 $t$ 检验比较 3 种湿地 5 种微地貌区之间的 $\mathrm{CH}_{4}$ 排放通量差异; 采用单因素多重配对Duncan分 析 $\mathrm{CH}_{4}$ 排放通量的季节性变化; 采用Pearson相关系 数评价 $\mathrm{CH}_{4}$ 排放通量与土壤温度、水位深度和地上 生物量的相关关系。所有数据采用SPSS 18.0软件包 进行分析; 图表中数据为平均值 \pm 标准偏差(mean \pm $S D)$ 。显著水平 $p=0.05$; 极显著水平 $p=0.01$ 。

\section{2 结果和分析}

\section{1 若尔盖高原湿地微地貌区 $\mathrm{CH}_{4}$ 排放通量的时 空变化}

若尔盖高原湿地5种微地貌区 $\mathrm{CH}_{4}$ 排放通量如 图1所示。2014年5-10月 $\mathrm{CH}_{4}$ 排放通量观测期间, 湿 地3种微地貌区(P-hummock、P-hollow和S-hummock) $\mathrm{CH}_{4}$ 排放通量存在极显著的季节性变化 $(p<$ 0.01), 而湿地Lawn和S-hollow两种微地貌区 $\mathrm{CH}_{4}$ 排 放通量无显著季节变化 $(p>0.05)$ 。P-hummock的 $\mathrm{CH}_{4}$ 排放通量曲线为单峰, 5 月初排放通量较低 (14.44 $\left.\mathrm{mg} \cdot \mathrm{m}^{-2} \cdot \mathrm{h}^{-1}\right)$, 随后大幅升高(32.73-56.52 $\left.\mathrm{mg} \cdot \mathrm{m}^{-2} \cdot \mathrm{h}^{-1}\right)$, 在9月初达到峰值 $\left(76.86 \mathrm{mg} \cdot \mathrm{m}^{-2} \cdot \mathrm{h}^{-1}\right)$,

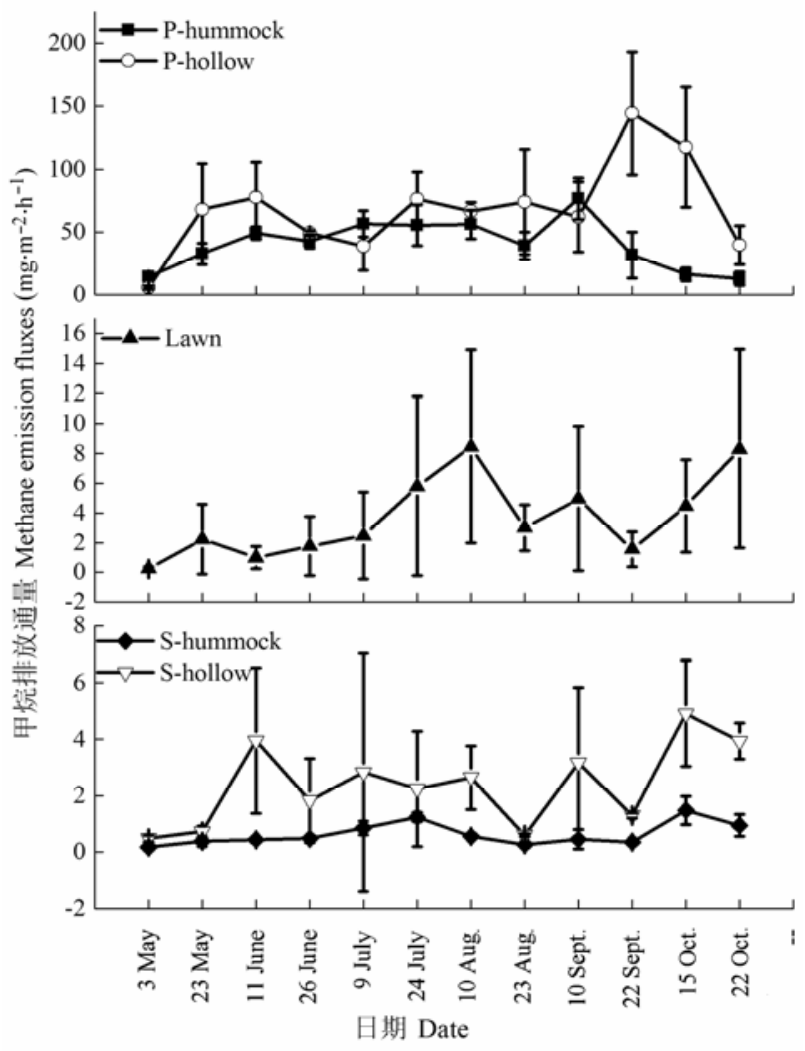

图1 若尔盖高原湿地5种微地貌区2014年 $\mathrm{CH}_{4}$ 排放通量季 节性变化(平均值土标准偏差)。Lawn, 常年性淹水与季节性 淹水点之间的过渡带平坦地; P-hollow, 常年性淹水洼地; P-hummock, 常年性淹水草丘; S-hollow, 季节性淹水洼地; S-hummock, 季节性淹水草丘。

Fig. 1 Seasonal variations of $\mathrm{CH}_{4}$ emission fluxes from Zoigê Plateau wetland on five microtopography in 2014 (mean $\pm S D$ ). Lawn, transitional zones between permanently flooded and seasonally flooded sites; P-hollow, permanently flooded hollow; P-hummock, permanently flooded hummock; S-hollow, seasonally flooded hollow; S-hummock, seasonally flooded hummock.

10月底达到最低值; P-hollow的 $\mathrm{CH}_{4}$ 排放通量曲线为 3 峰, 5 月初最低值为 $5.78 \mathrm{mg} \cdot \mathrm{m}^{-2} \cdot \mathrm{h}^{-1}$, 随后也大幅增 加，6月11日和7月24日出现2个小峰值，9月底达到 最大峰值 $\left(144.43 \mathrm{mg} \cdot \mathrm{m}^{-2} \cdot \mathrm{h}^{-1}\right), 10$ 月底回到较低水 平。S-hummock的 $\mathrm{CH}_{4}$ 排放通量曲线为双峰型, 5 月 初排放通量最低 $\left(0.17 \mathrm{mg} \cdot \mathrm{m}^{-2} \cdot \mathrm{h}^{-1}\right)$, 直到7月底达到 次峰值 $\left(1.24 \mathrm{mg} \cdot \mathrm{m}^{-2} \cdot \mathrm{h}^{-1}\right)$, 随后降低, 10月中旬达到 最大峰值 $\left(1.48 \mathrm{mg} \cdot \mathrm{m}^{-2} \cdot \mathrm{h}^{-1}\right)$; Lawn和S-hollow生长季 $\mathrm{CH}_{4}$ 排放通量无明显的季节变化, 曲线为双峰型, 峰值出现在6月初、8月初或秋末，排放通量范围分 别为 $0.23-8.45$ 和 $0.48-4.91 \mathrm{mg} \cdot \mathrm{m}^{-2} \cdot \mathrm{h}^{-1}$ 。

若尔盖高原湿地 5 种微地貌区 P-hummock、 P-hollow、Lawn、S-hummock、S-hollow生长季 $\mathrm{CH}_{4}$ 
排放通量分别为(40.32 \pm 19.78)、(68.48 \pm 36.23)、 $(3.68 \pm 2.73) 、(0.63 \pm 0.41) 、(2.38 \pm 1.45) \mathrm{mg} \cdot \mathrm{m}^{-2} \cdot \mathrm{h}^{-1}$ (平均值土标准偏差), 中值依次为 $40.81 、 67.51 、 2.74$ 、 0.46 和 $2.44 \mathrm{mg} \cdot \mathrm{m}^{-2} \cdot \mathrm{h}^{-1}$, 它们之间的 $\mathrm{CH}_{4}$ 排放通量平 均值存在显著差异 $(p<0.05)$ 。P-hollow的 $\mathrm{CH}_{4}$ 排放通 量最高, 是S-hummock的108倍, Lawn和S-hollow之 间的 $\mathrm{CH}_{4}$ 的排放通量倍数相差最小, 但也达1.6倍。 为了准确地预算区域 $\mathrm{CH}_{4}$ 排放量, 常年性淹水湿地 生长季 $\mathrm{CH}_{4}$ 排放通量(草丘和洼地面积比例55: 45)为 $(52.99 \pm 19.57) \mathrm{mg} \cdot \mathrm{m}^{-2} \cdot \mathrm{h}^{-1}$ (平均值土标准偏差); 季 节性淹水湿地生长季 $\mathrm{CH}_{4}$ 排放通量(草丘和洼地面 积比例 $55: 45)$ 为 $(1.42 \pm 0.82) \mathrm{mg} \cdot \mathrm{m}^{-2} \cdot \mathrm{h}^{-1}$ (平均值土标 准偏差)。

\section{2 若尔盖高原湿地微地貌区 $\mathrm{CH}_{4}$ 排放通量与土} 壤温度和水位深度的相关性

Pearson相关性分析表明P-hummock与5-30 cm 土壤温度显著相关 $(n=12, p<0.05)$ 或极显著相关 $(n$ $=12, p<0.01)$, 其他 4 种微地貌区 $\mathrm{CH}_{4}$ 排放与土壤温 度不显著相关 $(p>0.05)$, 但S-hummock剔除10月份 数据后, $\mathrm{CH}_{4}$ 排放通量与 $10-30 \mathrm{~cm}$ 土壤温度显著相 关 $(n=10, p<0.05)$ (表2)。5种微地貌区生长季 $\mathrm{CH}_{4}$ 排放通量与水位深度存在极显著线性正相关关系 $(n$ $=5, p<0.01)($ 表2)。

\section{3 讨论和结论}

\section{1 若尔盖高原湿地微地貌区 $\mathrm{CH}_{4}$ 排放通量的季} 节性变化

除了本研究湿地常年性淹水P-hollow $\mathrm{CH}_{4}$ 排放
通量范围(5.78-144.3 $\mathrm{mg} \cdot \mathrm{m}^{-2} \cdot \mathrm{h}^{-1}$ ) 稍大于其他研究 (见表3列出的参考文献)的高原湿地 $\mathrm{CH}_{4}$ 通量范围 $\left(-0.81-86.78 \mathrm{mg} \cdot \mathrm{m}^{-2} \cdot \mathrm{h}^{-1}\right)$ 以外, 其他 4 种微地貌区 $\mathrm{CH}_{4}$ 排放通量范围 $\left(0.17-76.86 \mathrm{mg} \cdot \mathrm{m}^{-2} \cdot \mathrm{h}^{-1}\right)$ 均在其他 研究的高原湿地 $\mathrm{CH}_{4}$ 通量范围内(表3)。

本研究湿地 5 种微地貌区 $\mathrm{CH}_{4}$ 排放通量峰值出 现在夏季(6-8月)或秋季(9-10月)(图1), 与以往研究 结果(Dise, 1993; Chen et al., 2008; 孙晓新等, 2009; 黄璞神等, 2011; Wei et al., 2015)一致。夏季 $\mathrm{CH}_{4}$ 排放 通量高的原因可能是夏季温度较高(图2), 促进湿地 植物的生长、分蔝, 为 $\mathrm{CH}_{4}$ 产生提供充足的有机底 物和传输通道(宋长春等, 2006; 孙晓新等, 2009; 黄 璞神等, 2011); 同时, 土壤微生物活性增强, 加快 土壤中氧的消耗, 降低了氧化还原电位, 有利于产 $\mathrm{CH}_{4}$ 菌的生长(孙晓新等, 2009; 黄璞神等, 2011)。秋 季 $\mathrm{CH}_{4}$ 排放通量高的原因可能是大量有机碳输入, 当年植物生长的根开始分解或调落物输入增加(孙 晓新等, 2009; 邓昭衡等, 2015), 使可利用活性有机 底物增加, 促进产 $\mathrm{CH}_{4}$ 菌产生 $\mathrm{CH}_{4}$; 另外, 秋季水位 深度增加(图2), 湿地土壤厌氧条件增多, 有利于产 $\mathrm{CH}_{4}$ 菌产生 $\mathrm{CH}_{4}$ 和减少氧化 $\mathrm{CH}_{4}$ 菌氧化 $\mathrm{CH}_{4}$ (Moore et al., 1994; 黄璞神等, 2011; Wei et al., 2015)。

然而, 湿地 $\mathrm{CH}_{4}$ 排放是土壤中 $\mathrm{CH}_{4}$ 的生成、氧 化、传输与释放过程相互作用的结果(Whalen \& Reeburgh, 1992; Le Mer \& Roger, 2001; 孙晓新等, 2009), 受到一系列因子(包含水位深度、温度、植物 和土壤性质等)影响, 使得生长季 $\mathrm{CH}_{4}$ 排放通量与土 壤温度和水位深度的相互关系更加复杂。本研究发

表2 $\mathrm{CH}_{4}$ 排放通量与土壤温度和水位深度的相关性

Table 2 Correlation between $\mathrm{CH}_{4}$ emission fluxes and soil temperature or water table depth

\begin{tabular}{|c|c|c|c|c|c|c|}
\hline $\begin{array}{l}\text { 样点 } \\
\text { Plot }\end{array}$ & $\begin{array}{l}\text { 回归方程 } \\
\text { Regression equation }\end{array}$ & $\begin{array}{l}\text { 变量 } \\
\text { Variable }\end{array}$ & $\begin{array}{l}\text { 变量范围 } \\
\text { Variable range }\end{array}$ & $R^{2}$ & $p$ & $n$ \\
\hline $\mathrm{CH}_{4}$ 排放通量平均值 Mean $\mathrm{CH}_{4}$ emission fluxes & $y=1.07 x+32.79$ & $W T D$ & $-39.7-29.5 \mathrm{~cm}$ & 0.919 & 0.006 & 5 \\
\hline \multirow[t]{5}{*}{ P-hummock } & $y=5.07 x-10.38$ & $T_{5}$ & $5.6-17.9{ }^{\circ} \mathrm{C}$ & 0.747 & 0.000 & 12 \\
\hline & $y=4.60 x-5.00$ & $T_{10}$ & $4.0-14.6{ }^{\circ} \mathrm{C}$ & 0.694 & 0.012 & 12 \\
\hline & $y=4.21 x-2.08$ & $T_{15}$ & $3.2-14.5{ }^{\circ} \mathrm{C}$ & 0.698 & 0.012 & 12 \\
\hline & $y=4.12 x-1.49$ & $T_{20}$ & $3.0-15.0{ }^{\circ} \mathrm{C}$ & 0.737 & 0.006 & 12 \\
\hline & $y=3.63 x+5.93$ & $T_{30}$ & $1.7-15.1{ }^{\circ} \mathrm{C}$ & 0.670 & 0.017 & 12 \\
\hline \multirow[t]{4}{*}{ S-hummock } & $y=0.07 x-0.27$ & $T_{10}$ & $4.0-14.6{ }^{\circ} \mathrm{C}$ & 0.407 & 0.028 & 10 \\
\hline & $y=0.07 x-0.23$ & $T_{15}$ & $3.2-14.5{ }^{\circ} \mathrm{C}$ & 0.448 & 0.020 & 10 \\
\hline & $y=0.06 x-0.19$ & $T_{20}$ & $3.0-15.0{ }^{\circ} \mathrm{C}$ & 0.429 & 0.024 & 10 \\
\hline & $y=0.05 x-0.07$ & $T_{30}$ & $1.7-15.1{ }^{\circ} \mathrm{C}$ & 0.346 & 0.043 & 10 \\
\hline
\end{tabular}

$T_{5}, 5 \mathrm{~cm}$ 土壤温度; $T_{10}, 10 \mathrm{~cm}$ 土壤温度; $T_{15}, 15 \mathrm{~cm}$ 土壤温度; $T_{20}, 20 \mathrm{~cm}$ 土壤温度; $T_{30}, 30 \mathrm{~cm}$ 土壤温度。WTD, 水位深度。

$T_{5}$, soil temperature at $5 \mathrm{~cm}$ depth; $T_{10}$, soil temperature at $10 \mathrm{~cm}$ depth; $T_{15}$, soil temperature at $15 \mathrm{~cm}$ depth; $T_{20}$, soil temperature at $20 \mathrm{~cm}$ depth; $T_{30}$, soil temperature at $30 \mathrm{~cm}$ depth. WTD, water table depth. 
表3 不同地区高原泥炭地生长季 $\mathrm{CH}_{4}$ 排放通量比较

Table 3 Comparison of $\mathrm{CH}_{4}$ emission fluxes in various plateau peatlands during the growing season

\begin{tabular}{|c|c|c|c|c|c|c|}
\hline $\begin{array}{l}\text { 位点 } \\
\text { Location }\end{array}$ & $\begin{array}{l}\text { 主要植被 } \\
\text { Main vegetation }\end{array}$ & $\begin{array}{l}\text { 水位深度 } \\
\text { water table } \\
\text { depth }(\mathrm{cm})\end{array}$ & $\begin{array}{l}\mathrm{CH}_{4} \text { 排放通量平均值 } \\
\text { Mean } \mathrm{CH}_{4} \mathrm{emission} \\
\text { fluxes }\left(\mathrm{mg} \cdot \mathrm{m}^{-2} \cdot \mathrm{h}^{-1}\right)\end{array}$ & $\begin{array}{l}\text { 范围 } \\
\text { Range } \\
\left(\mathrm{mg} \cdot \mathrm{m}^{-2} \cdot \mathrm{h}^{-1}\right)\end{array}$ & $\begin{array}{l}\text { 研究时间 } \\
\text { Study period }\end{array}$ & $\begin{array}{l}\text { 参考文献 } \\
\text { Reference }\end{array}$ \\
\hline $\begin{array}{l}\text { 若尔盖高原若尔盖县 } \\
\text { Zoigê Coonty of Zoigê Plateau }\end{array}$ & & & $23.10 \pm 30.28$ & $0.17-144.43$ & May to Oct. 2014 & $\begin{array}{l}\text { 本研究 } \\
\text { This study }\end{array}$ \\
\hline P-hollow & $\begin{array}{l}\text { 小眼子菜和狸藻 } \\
\text { Potamogeton pusillus and } \\
\text { Utricularia vulgaris }\end{array}$ & 29.5 & 68.48 & $5.78-144.43$ & & $\begin{array}{l}\text { 本研究 } \\
\text { This study }\end{array}$ \\
\hline P-hummock & $\begin{array}{l}\text { 木里蓋草 } \\
\text { Carex muliensis }\end{array}$ & 7.5 & 40.32 & $12.93-76.86$ & & $\begin{array}{l}\text { 本研究 } \\
\text { This study }\end{array}$ \\
\hline Lawn & $\begin{array}{l}\text { 西藏嵩草和花葶驴蹄草 } \\
\text { Kobresia tibetica and } \\
\text { Caltha scaposa }\end{array}$ & -21.6 & 3.68 & $0.23-8.45$ & & $\begin{array}{l}\text { 本研究 } \\
\text { This study }\end{array}$ \\
\hline S-hollow & $\begin{array}{l}\text { 木里薹草 } \\
\text { Carex muliensis }\end{array}$ & -21.1 & 2.38 & $0.48-4.91$ & & $\begin{array}{l}\text { 本研究 } \\
\text { This study }\end{array}$ \\
\hline S-hummock & $\begin{array}{l}\text { 西藏嵩草 } \\
\text { Kobresia tibetica }\end{array}$ & -39.7 & 0.63 & $0.17-1.48$ & & $\begin{array}{l}\text { 本研究 } \\
\text { This study }\end{array}$ \\
\hline $\begin{array}{l}\text { 若尔盖高原红原县 } \\
\text { Hongyuan County o }\end{array}$ & $\begin{array}{l}\text { 乌拉草 } \\
\text { Carex } m\end{array}$ & ND & 4.51 & $0.36-10.04$ & May to Sept. 2001 & Wang et al., 2002 \\
\hline 若尔盖 & 木里菨 & ND & 2.87 & $0.51-8.21$ & May to Sept. 2001 & Wang et al., 2002 \\
\hline $\begin{array}{l}\text { Hongyuan County of Zoigê Plateau } \\
\text { 若尔盖高原红原县 }\end{array}$ & $\begin{array}{l}\text { Carex muliensis } \\
\text { 乌拉草 }\end{array}$ & ND & 3.24 & $0.86-8.93$ & May to Oct. 2002 & Ding et al., 2004 \\
\hline $\begin{array}{l}\text { Hongyuan County of Zoigê Plateau } \\
\text { 若尔盖高原红原县 }\end{array}$ & $\begin{array}{l}\text { Carex meyeriana } \\
\text { 木里臺草 }\end{array}$ & ND & 1.24 & $0.16-5.75$ & May to Oct. 2002 & Ding et al., 2004 \\
\hline $\begin{array}{l}\text { Hongyuan County of Zoigê Plateau } \\
\text { 若尔盖高原红原县 }\end{array}$ & $\begin{array}{l}\text { Carex muliensis } \\
\text { 木里臺草和乌拉草 }\end{array}$ & ND & 2.43 & $0.02-12.01$ & May to Oct. 2003 & Wang, 2010 \\
\hline Hongyuan County of Zoigê Plateau & $\begin{array}{l}\text { Carex muliensis and Carex } \\
\text { meyeriana }\end{array}$ & & & & & \\
\hline $\begin{array}{l}\text { 若尔盖高原若尔盖县 } \\
\text { Zoigê County of Zoigê Plateau }\end{array}$ & $\begin{array}{l}\text { 西藏嵩草和木里薹草 } \\
\text { Kobresia tibetica and Carex } \\
\text { muliensis }\end{array}$ & $-18.36-10.66$ & 14.45 & $0.17-86.78$ & June to Sept. 2005 & Chen et al., 2008 \\
\hline $\begin{array}{l}\text { 若尔盖高原若尔盖县 } \\
\text { Zoigê County of Zoigê Plateau }\end{array}$ & $\begin{array}{l}\text { 木里臺草 } \\
\text { Carex muliensis }\end{array}$ & $-53.94--4.74$ & 9.83 & $0.06-39.5$ & June to Sept. 2009 & Li et al., 2011 \\
\hline 青藏高原 & 藏北嵩草和签草 & ND & $2.80 \pm 0.80$ & ND & July to Aug. 1996 & Wei et al., 2015 \\
\hline Qinghai-Xizang Plateau & $\begin{array}{l}\text { Kobresia littledalei and } \\
\text { Carex doniana }\end{array}$ & & & & & \\
\hline 青藏高原 & 毛柄水毛茛 & ND & 0.27 & $-0.81-2.64$ & April to Sept. 1997 & Jin et al., 1999 \\
\hline $\begin{array}{l}\text { Qinghai-Xizang Plateau } \\
\text { 青藏高原 }\end{array}$ & $\begin{array}{l}\text { Batrachium trichophyllum } \\
\text { 杉叶藻 }\end{array}$ & $10-120$ & 1.46 & $-0.24-7.85$ & April to Sept. 1997 & Jin et al., 1999 \\
\hline $\begin{array}{l}\text { Qinghai-Xizang Plateau } \\
\text { 青藏高原 }\end{array}$ & $\begin{array}{l}\text { Hippuris vulgaris } \\
\text { 臺草属 }\end{array}$ & 12 & 8.19 & $1.91-10.58$ & July to Sept. 2002 & Hirota et al., 2004 \\
\hline $\begin{array}{l}\text { Qinghai-Xizang Plateau } \\
\text { 青藏高原 }\end{array}$ & $\begin{array}{l}\text { Carex allivescers } \\
\text { 帕米尔臺草 }\end{array}$ & 0.2 & 5 & $2.88-6.91$ & May to Sept. 2012 & Song et al., 2015 \\
\hline $\begin{array}{l}\text { Qinghai-Xizang Plateau } \\
\text { 青藏高原 }\end{array}$ & $\begin{array}{l}\text { Carex pamirensis } \\
\text { 帕米尔薹草 }\end{array}$ & 0.6 & 6.11 & $4.61-13.25$ & May to Sept. 2013 & Song et al., 2015 \\
\hline $\begin{array}{l}\text { Qinghai-Xizang Plateau } \\
\text { 美国科罗拉多州弗兰特山脉 }\end{array}$ & $\begin{array}{l}\text { Carex pamirensis } \\
\text { 臺草 }\end{array}$ & ND & 0.35 & $0.05-1.10$ & June to Sept. 1992 & West et al., 1999 \\
\hline $\begin{array}{l}\text { Colorado Front Range, USA } \\
\text { 北美洲落基山脉 } \\
\text { Rocky Mountains, North America }\end{array}$ & $\begin{array}{l}\text { Carex scopulorum } \\
\text { 臺草 } \\
\text { Carex aquatilis }\end{array}$ & ND & 11.45 & $0.04-20.41$ & May to Oct.1996 & $\begin{array}{l}\text { Wickland et al., } \\
1999\end{array}$ \\
\hline
\end{tabular}

ND, 无有效数据。Lawn, 常年性淹水与季节性淹水点之间的过渡带平坦地; P-hollow, 常年性淹水洼地; P-hummock, 常年性淹水草丘; S-hollow, 季节 性淹水洼地; S-hummock, 季节性淹水草丘。

ND, no data available. Lawn, transitional zones between permanently flooded and seasonally flooded sites; P-hollow, permanently flooded hollow; P-hummock, permanently flooded hummock; S-hollow, seasonally flooded hollow; S-hummock, seasonally flooded hummock.

现湿地微地貌区(P-hummock、P-hollow和S-hummock) $\mathrm{CH}_{4}$ 排放通量季节性变化存在极显著差异 $(p$ $<0.01)$, Lawn和S-hollow $\mathrm{CH}_{4}$ 排放通量季节变化不 显著 $(p>0.05)$ 。Pearson相关性分析表明整个生长季 仅发现常年性淹水P-hummock与土壤温度(5-30 $\mathrm{cm})$ 存在显著线性正相关关系 $(p<0.05)$ (表2), 这表 明土壤温度是影响该微地貌区 $\mathrm{CH}_{4}$ 排放通量存在显 著季节变化的主控因子，与其他报道的北方湿地 $\mathrm{CH}_{4}$ 排放与温度显著相关通常局限于常年性淹水湿
地(Whalen \& Reeburgh, 1992; 孙晓新等, 2009)吻 合。但常年性淹水P-hollow没有发现这一规律, 说明 还有其他因子影响湿地 $\mathrm{CH}_{4}$ 排放, 诸如水位深度和 植物类型。常年性淹水P-hollow整个生长季水位深 度超过土壤地表20 cm (图2); 另外, 植被类型以沉 水植物小眼子菜和狸藻为主要植物群落, 它们不像 维管植物通气组织(尤其是莎草科)的传输促进了 $\mathrm{CH}_{4}$ 从土壤向大气的输送, 进而增加 $\mathrm{CH}_{4}$ 排放 (McEwing et al., 2015), 如P-hummock样点以莎草科 

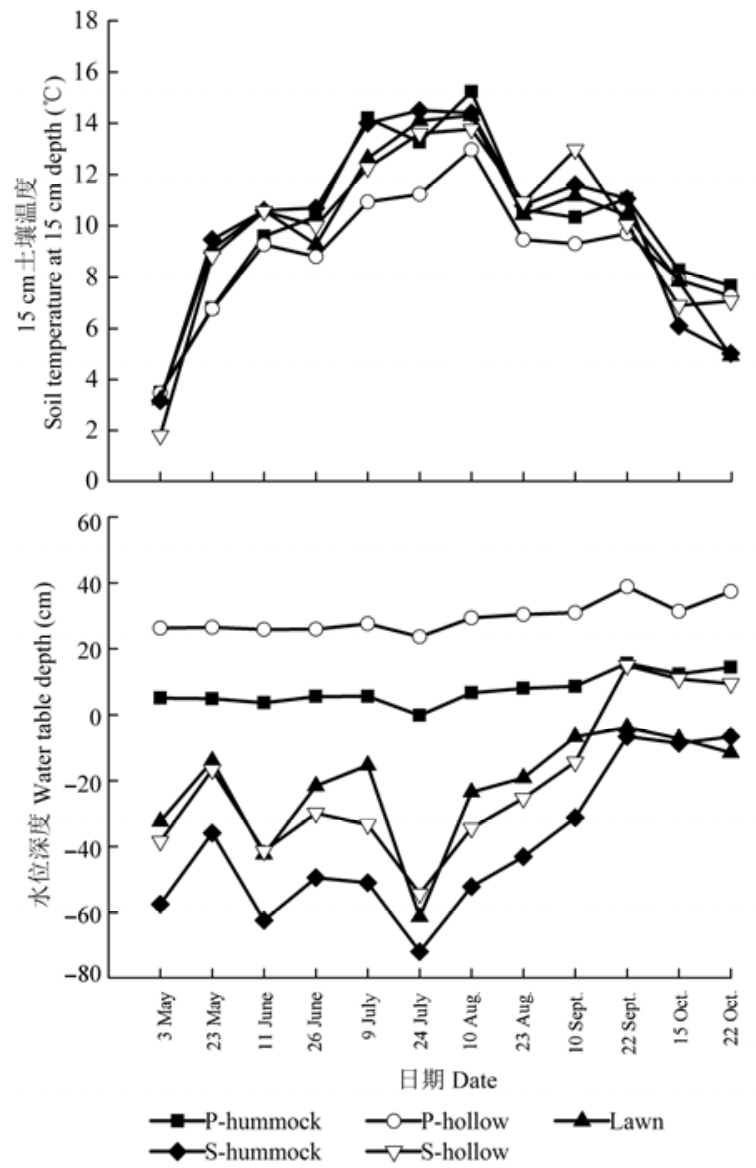

图2 2014年若尔盖高原湿地5种微地貌区15 $\mathrm{cm}$ 土壤温度和 水位深度。Lawn, 常年性淹水与季节性淹水点之间的过渡带 平坦地; P-hollow, 常年性淹水洼地; P-hummock, 常年性淹 水草丘; S-hollow, 季节性淹水洼地; S-hummock, 季节性淹 水草丘。

Fig. 2 Soil temperature at $15 \mathrm{~cm}$ depth and water table depth from the Zoige Plateau wetland on five microtopography in 2104. Lawn, transitional zones between permanently flooded and seasonally flooded sites; P-hollow, permanently flooded hollow; P-hummock, permanently flooded hummock; S-hollow, seasonally flooded hollow; S-hummock, seasonally flooded hummock.

植物木里薹草为优势种。这种水位深度和植被类型 因子的变化, 可能使湿地土壤-大气 $\mathrm{CH}_{4}$ 交换方式发 生改变。许多研究表明湖泊湿地或泥炭湿地淹水小 池塘水-大气界面 $\mathrm{CH}_{4}$ 交换方式以冒泡式(ebullition) 为主 $(>95 \%)$ (Keller \& Stallard, 1994; Casper et al., 2000), 其 $\mathrm{CH}_{4}$ 排放通量极高(Keller \& Stallard, 1994; Mikkelä et al., 1995), 进而影响区域 $\mathrm{CH}_{4}$ 排放预算 (Walter et al., 2006; Tokida et al., 2007), 这可能导致 $\mathrm{CH}_{4}$ 排放通量与温度或水位深度的关系趋于复杂。 例如, 在9月底和10月观测到P-hollow $\mathrm{CH}_{4}$ 排放通量 的最高值为 592.44 和 $327.82 \mathrm{mg} \cdot \mathrm{m}^{-2} \cdot \mathrm{h}^{-1}$ 。在北极圈河
流阶地水淹洼地 $\mathrm{CH}_{4}$ 排放通量的最高值为 559 $\mathrm{mg} \cdot \mathrm{m}^{-2} \cdot \mathrm{h}^{-1}$ (van Huissteden et al., 2005)。北极圈湖泊 解冻后, 冒泡式 $\mathrm{CH}_{4}$ 排放通量达到 $300 \mathrm{mg} \cdot \mathrm{m}^{-2} \cdot \mathrm{h}^{-1}$ (Walter et al., 2006)。S-hummock生长季 $\mathrm{CH}_{4}$ 排放通 量随温度增加而逐渐增加, 7月底出现次峰(图1), 秋 季水位深度升高(图2)和调落物输入, 出现最大峰 值, 可能掩盖了温度对 $\mathrm{CH}_{4}$ 排放的作用。剔除10月 份数据后, S-hummock $\mathrm{CH}_{4}$ 排放通量与土壤(10-30 $\mathrm{cm}$ )温度显著线性正相关(表2), 暗示秋季水位深度 升高或调落物输入促进了湿地 $\mathrm{CH}_{4}$ 排放。

Lawn和S-hollow $\mathrm{CH}_{4}$ 排放通量无明显的季节变 化, 这可能与该微地貌区水位深度(平均值为-21.6 $\mathrm{cm}$ 和 $-21.1 \mathrm{~cm}$ )条件下的产 $\mathrm{CH}_{4}$ 菌和氧化 $\mathrm{CH}_{4}$ 菌的竞 争有关(Hirota et al., 2004; Sun et al., 2011)。通常湿 地土壤表层 $\mathrm{CH}_{4}$ 氧化潜力较大(王长科等, 2004; Lai, 2009), 水位深度下降后, $\mathrm{CH}_{4}$ 氧化加强, $\mathrm{CH}_{4}$ 排放通 量降低, 导致无明显高 $\mathrm{CH}_{4}$ 排放通量, 可能使得观 测期间 $\mathrm{CH}_{4}$ 排放通量无明显的季节变化(Sun et al., 2011)。水位深度下降还导致下层土壤温度增加, 进 而促进产 $\mathrm{CH}_{4}$ 菌生成 $\mathrm{CH}_{4}$, 促进了 $\mathrm{CH}_{4}$ 排放。在这两 种微地貌区观测期间 $\mathrm{CH}_{4}$ 排放变异系数较大 (7.2\%-149.3\%, 变异系数 $>40 \%$ 的比例占 $75 \%$ )(图1), 进而推测标准偏差过大可能也使得 $\mathrm{CH}_{4}$ 排放通量无 明显的季节性变化, 这与Mikkelä等(1995)研究发现 的北方泥炭地微地貌区小池塘 $\mathrm{CH}_{4}$ 排放通量无明显 日变化格局的结果类似。但是, 这两种微地貌区在 夏季或秋季具有较高 $\mathrm{CH}_{4}$ 排放通量(图1), 说明温 度、水位深度和调落物输入对湿地 $\mathrm{CH}_{4}$ 排放影响较 大。

\section{2 若尔盖高原湿地微地貌区 $\mathrm{CH}_{4}$ 排放通量的空} 间变化

若尔盖高原湿地 5 种微地貌区 $\mathrm{CH}_{4}$ 排放通量大 小顺序为：P-hollow > P-hummock > Lawn> S-hollow > S-hummock, 这与其他研究的湿地不同 微地貌区 $\mathrm{CH}_{4}$ 排放通量规律吻合, 即水位深度较高 的微地貌区 $\mathrm{CH}_{4}$ 排放通量较高(Clymo et al., 1995; Kutzbach et al., 2004; Glagolev et al., 2011; Wei et al., 2015)。许多研究表明不同湿地生态系统内, 特 别是泥炭地微地貌区的 $\mathrm{CH}_{4}$ 排放通量存在显著的空 间变化(Moore et al., 1994; Clymo et al., 1995; Mikkelä et al., 1995; Waddington \& Roulet, 1996; Glagolev \& Shnyrev, 2008; Kalyuzhnyi et al., 2009; 
Glagolev et al., 2011; Munir \& Strack, 2014)。本研究 湖滨湿地的 5 种微地貌区之间 $\mathrm{CH}_{4}$ 排放通量的平均 值存在显著的空间差异性(表3), 变异系数为 $131 \%$ 。 Pearson相关性分析表明5种微地貌区 $\mathrm{CH}_{4}$ 排放通量 的平均值与水位深度平均值存在极显著的线性正 相关关系(表2), 表明影响该湖滨湿地微地貌区 $\mathrm{CH}_{4}$ 排放通量存在空间差异的主控因子是水位深度, 这 与其他报道的北方湿地的研究结果(Moore et al., 1994; Ding et al., 2003; Huttunen et al., 2003) 一致。 究其原因: 一方面可能是草丘(hummock)的水位深 度较低, 产 $\mathrm{CH}_{4}$ 菌生成的 $\mathrm{CH}_{4}$ 大部分被氧化(Moore et al., 1994; Clymo et al., 1995; Lai, 2009; Wei et al., 2015), 进而减少了 $\mathrm{CH}_{4}$ 排放; 另一方面, 洼地 $\mathrm{CH}_{4}$ 排放通量高于草丘, 可能是由于较高水位深度, 产 $\mathrm{CH}_{4}$ 菌生成 $\mathrm{CH}_{4}$ 和温度升高后, 促进了 $\mathrm{CH}_{4}$ 排放 (Waddington \& Roulet, 1996; Lai, 2009; Wei et al., 2015), 这表明水位深度在调控湿地 $\mathrm{CH}_{4}$ 排放通量中 发挥着极其重要的作用。如果不考虑这种差异, 可 能会低估或高估区域 $\mathrm{CH}_{4}$ 排放量。例如, P-hollow $\mathrm{CH}_{4}$ 排放通量最高, 它是S-hummock $\mathrm{CH}_{4}$ 最低排放 通量的108倍, Lawn和S-hollow $\mathrm{CH}_{4}$ 排放通量倍数相 差最小, 也达1.6倍。因此, 研究湿地生态系统内部 微地貌区 $\mathrm{CH}_{4}$ 排放特征具有重要意义, 量化其数值, 进一步通过与高精度分辨率的遥感或地理信息系 统数据结合, 将有助于精确地预算若尔盖高原湿地 的 $\mathrm{CH}_{4}$ 排放量。

致谢 本研究得到中国清洁发展机制基金赠款项目 (2012076)和中国林业科学研究院林业新技术研究 所基本科研业务费专项(CAFINT2014K06)的资助。 若尔盖高寒湿地生态系统定位研究站和若尔盖湿地 国家级自然保护区管理局对本研究给予大力支持和 帮助, 在此特别感谢。

\section{参考文献}

Casper P, Maberly SC, Hall GH, Finlay BJ (2000). Fluxes of methane and carbon dioxide from a small productive lake to the atmosphere. Biogeochemistry, 49, 1-19.

Chen H, Yao SP, Wu N, Wang YF, Luo P, Tian JQ, Gao YH, Sun $G$ (2008). Determinants influencing seasonal variations of methane emissions from alpine wetlands in Zoigê Plateau and their implications. Journal of Geophysical Research, 113(D12), doi:10.1029/2006JD008072.

Chen YH, Prinn RG (2006). Estimation of atmospheric methane emissions between 1996 and 2001 using a threedimensional global chemical transport model. Journal of
Geophysical Research, 111(D10), doi:10.1029/2005JD006058.

Clymo RS, Pearce DME, Conrad R (1995). Methane and carbon dioxide production in, transport through, and efflux from a peatland [and discussion]. Philosophical Transactions of the Royal Society A: Mathematical, Physical and Engineering Sciences, 351, 249-259.

Deng ZH, Zhang XW, Gao JJ, Gao JQ (2015). Effects of soil moisture and litter on $\mathrm{CH}_{4}$ emission from peat in Zoigê wetlands. Journal of Ecology and Rural Environment, 31, 548-552. (in Chinese with English abstract) [邓昭衡, 张 雪雯, 高居娟, 高俊琴 (2015). 水分及调落物对若尔盖 泥炭土 $\mathrm{CH}_{4}$ 排放的影响. 生态与农村环境学报, 31 , 548-552.]

Ding WX, Cai ZC (2002). Methane emission from mires and its influencing factors. Scientia Geographica Sinica, 22, 619-625. (in Chinese with English abstract) [丁维新, 蔡 祖聪 (2002). 沼泽甲烷排放及其主要影响因素. 地理科 学, 22, 619-625.]

Ding WX, Cai ZC, Tsuruta H, Li XP (2003). Key factors affecting spatial variation of methane emissions from freshwater marshes. Chemosphere, 51, 167-173.

Ding WX, Cai ZC, Wang DX (2004). Preliminary budget of methane emissions from natural wetlands in China. Atmospheric Environment, 38, 751-759.

Dise NB (1993). Methane emission from Minnesota peatlands: Spatial and seasonal variability. Global Biogeochemical Cycles, 7, 123-142.

Glagolev M, Kleptsova I, Filippov I, Maksyutov S, Machida T (2011). Regional methane emission from West Siberia mire landscapes. Environmental Research Letters, 6, doi:10.1088/1748-9326/6/4/045214.

Glagolev MV, Shnyrev NA (2008). Methane emission from mires of Tomsk oblast in the summer and fall and the problem of spatial and temporal extrapolation of the obtained data. Moscow University Soil Science Bulletin, 63(2), 67-80.

Hirota M, Tang YH, Hu QW, Hirata S, Kato T, Mo WH, Cao GM, Mariko S (2004). Methane emissions from different vegetation zones in a Qinghai-Tibetan Plateau wetland. Soil Biology \& Biochemistry, 36, 737-748.

Huang PY, Yu HX, Chai LH, Chai FY, Zhang WF (2011). Methane emission flux of Zhalong Phragmites australis wetlands in growth season. Chinese Journal of Applied Ecology, 22, 1219-1224. (in Chinese with English abstract) [黄璞神, 于洪贤, 柴龙会, 柴方营, 张万峰 (2011). 扎龙芦苇湿地生长季的甲烷排放通量. 应用生 态学报, 22, 1219-1224.]

Huttunen JT, Nykänen H, Turunen J, Martikainen PJ (2003). Methane emissions from natural peatlands in the northern boreal zone in Finland, Fennoscandia. Atmospheric Environment, 37, 147-151. 
Inubushi K, Otake S, Furukawa Y, Shibasaki N, Ali M, Itang AM, Tsuruta H (2005). Factors influencing methane emission from peat soils: Comparison of tropical and temperate wetlands. Nutrient Cycling in Agroecosystems, 71, 93-99.

IPCC (Intergovernmental Panel on Climate Change) (2013). Climate change 2013: The physical science basis. In: Stocker TF, Qin D, Plattner GK, Tignor M, Allen SK, Boschung J, Nauels A, Xia Y, Bex V, Midgley PM eds. Contribution of Working Group I to the Fifth Assessment Report of the Intergovernmental Panel on Climate Change. Cambridge University Press, Cambridge, UK.

Jin HJ, Wu J, Cheng GD, Nakano T, Sun GY (1999). Methane emissions from wetlands on the Qinghai-Tibet Plateau. Chinese Science Bulletin, 44, 2282-2286.

Kalyuzhnyi IL, Lavrov SA, Reshetnikov AI, Paramonova NN, Privalov VI (2009). Methane emission from the oligotrophic bog massif in the Northwestern Russia. Russian Meteorology and Hydrology, 34, 35-45.

Keller M, Stallard RF (1994). Methane emission by bubbling from Gatun Lake, Panama. Journal of Geophysical Research, 99(D4), 8307-8319.

Kirschke S, Bousquet P, Ciais P, Saunois M, Canadell JG, Dlugokencky EJ, Bergamaschi P, Bergmann D, Blake DR, Bruhwiler L, Cameron-Smith P, Castaldi S, Chevallier F, Feng L, Fraser A, Heimann M, Hodson EL, Houweling S, Josse B, Fraser PJ, Krummel PB, Lamarque JF, Langenfelds RL, Le Quéré C, Naik V, O’Doherty S, Palmer PI, Pison I, Plummer D, Poulter B, Prinn RG, Rigby M, Ringeval B, Santini M, Schmidt M, Shindell DT, Simpson IJ, Spahni R, Steele LP, Strode SA, Sudo K, Szopa S, van der Werf GR, Voulgarakis A, van Weele M, Weiss RF, Williams JE, Zeng G (2013). Three decades of global methane sources and sinks. Nature Geoscience, 6, 813823.

Kutzbach L, Wagner D, Pfeiffer EM (2004). Effect of microrelief and vegetation on methane emission from wet polygonal tundra, Lena Delta, Northern Siberia. Biogeochemistry, 69, 341-362.

Lai DYF (2009). Methane dynamics in northern peatlands: A review. Pedosphere, 19, 409-421.

Le Mer J, Roger P (2001). Production, oxidation, emission and consumption of methane by soils: A review. European Journal of Soil Biology, 37, 25-50.

Li L, Lei GC, Gao JQ, Lu C, Zhou Y, Jia YF, Yang M, Suolang DEJ (2011). Effect of water table and soil water content on methane emission flux at Carex muliensis marshes in Zoigê Plateau. Wetland Science, 9(2), 173-178. (in Chinese with English abstract) [李丽, 雷光春, 高俊琴, 吕 偲, 周延, 贾亦飞, 杨萌, 索郎夺尔基 (2011). 地下水 位和土壤含水量对若尔盖木里薹草沼泽甲烷排放通量 的影响. 湿地科学, 9(2), 173-178.]

Mastepanov M, Sigsgaard C, Dlugokencky EJ, Houweling S,
Ström L, Tamstorf MP, Christensen TR (2008). Large tundra methane burst during onset of freezing. Nature, 456, 628-630.

McEwing KR, Fisher JP, Zona D (2015). Environmental and vegetation controls on the spatial variability of $\mathrm{CH}_{4}$ emission from wet-sedge and tussock tundra ecosystems in the Arctic. Plant and Soil, 388, 37-52.

Mikkelä C, Sundh I, Svensson BH, Nilsson M (1995). Diurnal variation in methane emission in relation to the water table, soil temperature, climate and vegetation cover in a Swedish acid mire. Biogeochemistry, 28, 93-114.

Moore TR, Heyes A, Roulet NT (1994). Methane emissions from wetlands, southern Hudson Bay lowland. Journal of Geophysical Research, 99(D1), 1455-1467.

Munir TM, Strack M (2014). Methane flux influenced by experimental water table drawdown and soil warming in a dry boreal continental bog. Ecosystems, 17, 1271-1285.

Rigby M, Prinn RG, Fraser PJ, Simmonds PG, Langenfelds RL, Huang J, Cunnold DM, Steele LP, Krummel PB, Weiss RF, O’Doherty S, Salameh PK, Wang HJ, Hart CM, Mühle J, Porter LW (2008). Renewed growth of atmospheric methane. Geophysical Research Letters, 35, doi:10. 1029/2008GL036037.

Song CC, Zhang LH, Wang YY, Zhao ZC (2006). Annual dynamics of $\mathrm{CO}_{2}, \mathrm{CH}_{4}, \mathrm{~N}_{2} \mathrm{O}$ emissions from freshwater marshes and affected by nitrogen fertilization. Environmental Science, 7, 2369-2375. (in Chinese with English abstract) [宋长春, 张丽华, 王毅勇, 赵志春 (2006). 淡 水沼泽湿地 $\mathrm{CO}_{2} 、 \mathrm{CH}_{4}$ 和 $\mathrm{N}_{2} \mathrm{O}$ 排放通量年际变化及其对 氮输入的响应. 环境科学, 27, 2369-2375.]

Song WM, Wang H, Wang GS, Chen LT, Jin ZN, Zhuang QL, He JS (2015). Methane emissions from an alpine wetland on the Tibetan Plateau: Neglected but vital contribution of the nongrowing season. Journal of Geophysical Research, 120,1475-1490.

Sun XX, Mu CC, Shi LY, Chen W, Liu X, Wu YX, Feng DJ (2009). Methane emission from forested swamps in Xiaoxing'an Mountains, northeastern China. Chinese Journal of Plant Ecology, 33, 535-545. (in Chinese with English abstract) [孙晓新, 牟长城, 石兰英, 程伟, 刘霞, 吴云霞, 冯登军 (2009). 小兴安岭森林沼泽甲烷排放及 其影响因子. 植物生态学报, 33, 535-545.]

Sun XX, Mu CC, Song CC (2011). Seasonal and spatial variations of methane emissions from montane wetlands in Northeast China. Atmospheric Environment, 45, 18091816

Tokida T, Miyazaki T, Mizoguchi M, Nagata O, Takakai F, Kagemoto A, Hatano R (2007). Falling atmospheric pressure as a trigger for methane ebullition from peatland. Global Biogeochemical Cycles, 21, doi:10.1029/2006GB002790.

van Huissteden J, Maximov TC, Dolman AJ (2005). High 
methane flux from an arctic floodplain (Indigirka lowlands, eastern Siberia). Journal of Geophysical Research, 110, G02002, doi:10.1029/2005JG000010.

Waddington JM, Roulet NT (1996). Atmosphere-wetland carbon exchanges: Scale dependency of $\mathrm{CO}_{2}$ and $\mathrm{CH}_{4}$ exchange on the developmental topography of a peatland. Global Biogeochemical Cycles, 10, 233-245.

Walter KM, Zimov SA, Chanton JP, Verbyla D, Chapin FS (2006). Methane bubbling from Siberian thaw lakes as a positive feedback to climate warming. Nature, 443, 71-75.

Wang CK, Lu XG, Zhou HR, Cai ZC, Luo Y (2004). Studies on methane oxidation by bog soils in Zoigê Plateau. China Environmental Science, 24, 646-649. (in Chinese with English abstract) [王长科, 吕宪国, 周华荣, 蔡祖聪, 罗 勇 (2004). 若尔盖高原沼泽土壤氧化甲烷的研究. 中国 环境科学, 24, 646-649.]

Wang DX (2010). Emission fluxes of carbon dioxide, methane and nitrous oxide from peat marsh in Zoigê Plateau. Wetland Science, 8(3), 220-224. (in Chinese with English abstract) [王德宣 (2010). 若尔盖高原泥炭沼泽二氧化碳、 甲烷和氧化亚氮排放通量研究. 湿地科学, 8(3), 220224.]

Wang DX, Lu XG, Ding WX, Cai ZC, Gao JF, Yang FM (2002). Methane emission from marshes in Zoigê Plateau. Advance in Earth Sciences, 17, 877-880. (in Chinese with English abstract) [王德宣, 吕宪国, 丁维新, 蔡祖聪, 高 景福, 杨福明 (2002). 若尔盖高原沼泽湿地 $\mathrm{CH}_{4}$ 排放研
究. 地球科学进展, 17, 877-880.]

Wang ZP, Duan Y, Yang JR, Chen QS, Han XG (2003). Spatial distribution of potential $\mathrm{CH}_{4}$ oxidation and production in Zoigê marsh of Qinghai-Tibet Plateau. Acta Phytoecologica Sinica, 27, 786-791. (in Chinese with English abstract) [王智平, 段毅, 杨居荣, 陈全胜, 韩兴国 (2003). 青藏高原若尔盖沼泽潜在 $\mathrm{CH}_{4}$ 氧化与生成的分 布特征. 植物生态学报, 27, 786-791.]

Wei D, Xu R, Tarchen T, Dai DX, Wang YS, Wang YH (2015). Revisiting the role of $\mathrm{CH}_{4}$ emissions from alpine wetlands on the Tibetan Plateau: Evidence from two in situ measurements at 4758 and $4320 \mathrm{~m}$ above sea level. Journal of Geophysical Research, 120, 1741-1750.

West AE, Brooks PD, Fisk MC, Smith LK, Holland EA, Jaeger CH, Babcock S, Lai RS, Schmidt SK (1999). Landscape patterns of $\mathrm{CH}_{4}$ fluxes in an alpine tundra ecosystem. Biogeochemistry, 45, 243-264.

Whalen SC, Reeburgh WS (1992). Interannual variations in tundra methane emission: A 4-year time series at fixed sites. Global Biogeochemical Cycles, 6, 139-159.

Wickland KP, Striegl RG, Schmidt SK, Mast MA (1999). Methane flux in subalpine wetland and unsaturated soils in the southern Rocky Mountains. Global Biogeochemical Cycles, 13, 101-113.

责任编委: 倪 健 责任编辑: 王 葳

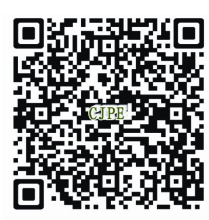

植物生态学报官网

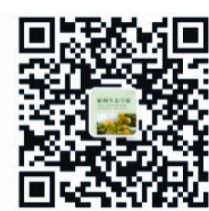

微信订阅号

期刊及学科

相关信息发布

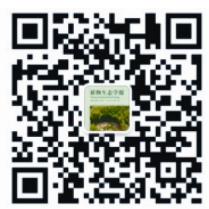

微信服务号

稿件状态查询 全文检索汶览 